\title{
Long Term Investment Optimization Methodology for Multi-Zonal Transmission Expansion
}

\author{
Hakan Ergun \\ Ronnie Belmans \\ Dirk Van Hertem \\ University of Leuven \\ Dept. of electrical engineering, Div. ELECTA \\ Leuven, Belgium
}

\author{
Barry Rawn \\ Brunel University \\ Dept. of Electronic and Computer Engineering \\ College of Engineering, Design and Physical Sciences \\ London, UK
}

\begin{abstract}
This paper introduces a stepwise investment optimization methodology for transmission system expansion planning. The objective of the developed methodology is to determine transmission expansion plans to realize a desired interconnection capacity between multiple zones minimizing investment and operational costs. The methodology uses MILP optimization and a modified $A^{*}$ shortest path algorithm sequentially in order to determine the optimal investment time point, transmission topology, technology and routing. Spatial constraints and their effects on the installation cost are taken into account in the technology and route optimization. A possible application of the methodology is demonstrated on a stepwise investment plan for the North Sea region.
\end{abstract}

Index Terms-Transmission Expansion Planning, Transmission System Investments, Investment Optimization, Optimal Routing, North Sea

\section{INTRODUCTION}

The electricity sector is currently facing major changes and challenges around the globe. There is a trend to use renewable energy sources for power generation in order to decrease greenhouse gas emissions. The increased use of renewable energy sources puts the transmission system under stress due to increased power flow and power flow volatility. This makes significant investments in transmission system infrastructure inevitable. The European Network of Transmission System Operators (ENTSO-E) stimates that $52300 \mathrm{~km}$ of new or refurbished extra high voltage routes will be required in the coming decade, equal to an investment volume of 104 billion $€[1]$.

The use of optimization tools can help to achieve significant cost savings in infrastructure investments of such volumes. As transmission grid planning over long time horizons is subjected to a large number of uncertainties, robust optimization methodologies need to be developed. These methods must be computationally tractable allowing the analysis of many different scenarios and helping to minimize investment risks.

A large number of transmission system investment optimization methodologies are described in the literature [2]-[4]. These methodologies tackle the transmission system investment problem from different angles. A key short-coming of available planning methodologies is that spatial aspects and the use of different technology options for new investments are not taken into account, especially in dynamic or pseudo-dynamic planning methodologies. This work provides a methodology to determine optimal time points, rating, location, routing and technology of new transmission investments between multiple zones. The output of the methodology is a detailed and optimized stepwise investment plan for a defined planning horizon. The developed methodology can be useful to transmission system operators to efficiently analyze different future scenarios in order and reduce the risk of future investments.

This paper extends the optimization methodologies presented in [5], [6] to multiple transmission zones. Additionally, transmission losses have been introduced in the optimization in order to better assess the total life cycle costs and better quantify the transmission losses of different technology options. A possible application of the developed methodology is shown on the example of the North Sea region.

\section{Methodology}

The developed optimization methodology uses interconnection capacities, $P_{t}^{\text {inter }}$ as input, which can be provided by a market model using a simplified grid model (fig. 1 interconnection level). Using the generator cost information, a probabilistic optimal load flow calculation is performed on a detailed grid model (fig. 1 - network abstraction). The outcome of the network abstraction are the maximum power injection capabilities, $\vec{P}_{M P I C, t}$ indicating how much power can be injected in each node of the grid without causing overload situations. $\tilde{\Delta}_{M P I C}^{\text {tot }}$ describes the change in the maximum power injection capabilities depending on injections in other nodes of the system, as an injection in any node of the system will change the maximum power injection capability of all other nodes. $\tilde{\Delta}_{M P I C}^{t o t}$ is calculated between all nodes of the system. As a linear DC optimal power flow approach is used, the change in maximum power capabilities can be approximated by sum of $\tilde{\Delta}_{M P I C}^{t o t}$ values, in case more than one injection occurs. The calculation methodology for $\vec{P}_{M P I C, t}$ and is described in greater detail in [7], [8].

The third block of the optimization methodology determines the optimal time point, rating, technology and transmission route for new investments in order to satisfy the total interzonal transmission capacity $P_{t}^{\text {inter }}$ for each time point of the 


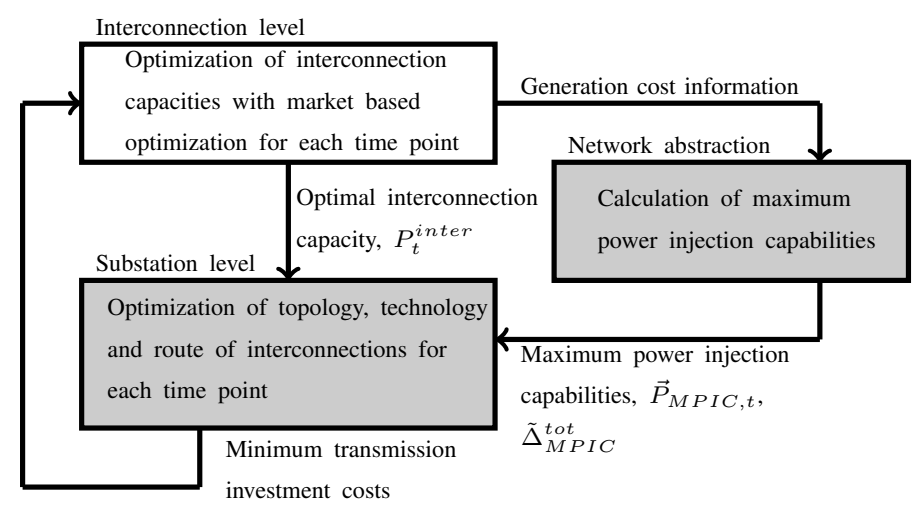

Figure 1: Structure of the proposed long term transmission expansion optimization methodology [6]

planning horizon. The objective of the optimization is the minimization of total system costs, consisting of equipment price, installation cost and transmission losses. The optimization problem is solved by iteratively using an MILP optimization and an optimal routing algorithm as depicted in fig. 2. The following sections describe steps 1-3 of fig. 2

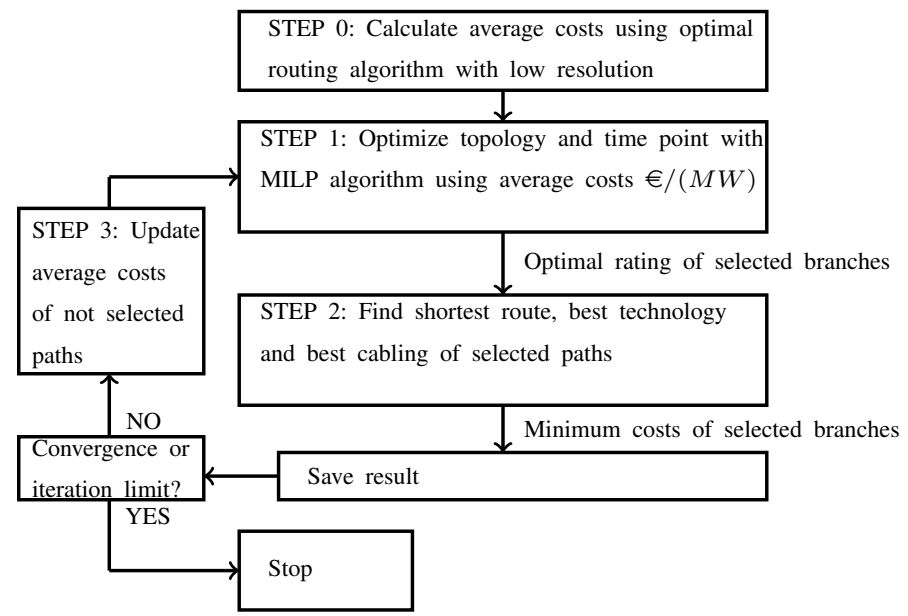

Figure 2: Iterative optimization methodology using MILP and optimal routing algorithm [6]

\section{A. MILP Optimization}

The objective of the optimization problem is the minimization of transmission system costs over the entire planning horizon consisting of cost of investment, installation and losses. The optimization delivers the investment time point and power rating of future investments. Equation (1) shows the objective function of the MILP problem statement. The search space consists of $N_{s}=3 \cdot N_{b} \cdot N_{k} \cdot N_{t}$ binary decision variables, where $N_{b}$ is the number of possible branches, $N_{k}$ the number of possible power ratings and $N_{t}$ is the number of investment time points. $U_{i j t}$ is a binary decision variable defined for each possible branch $i$, power rating $j$ and time point $t$. For each branch, rating and time point 3 variables are defined. The first binary variable is the investment variable $U_{i j t}^{i n v}$, stating if the branch is built or not. The other variables $U_{i j t}^{+}$and $U_{i j t}^{-}$account for the power flows on the lines in positive and negative direction.

$$
\begin{aligned}
\min _{\substack{\forall U^{i n v}, U^{+}, U^{-} \\
\in B^{N_{b} \times N_{k} \times N_{t}}}} C_{\text {cap }} & =\left(U^{i n v}+U^{+}+U^{-}\right):(\overrightarrow{1} \otimes \mathbf{k}):(\overrightarrow{1} \otimes \mathbf{t}) \cdot \tilde{C} \\
& =\sum_{i} \sum_{j} \sum_{t}\left(U_{i j t}^{i n v} U_{i j t}^{+}+U_{i j t}^{-}\right) \cdot K_{i j t} \cdot \tilde{C}_{i j t}
\end{aligned}
$$

Vector $\mathbf{k}$ contains all possible power ratings of transmission paths. Vector $\tilde{C}$ contains costs for each transmission path, power rating and investment time point. For instance $C_{2,5,3}$ is the investment costs of path 2 , using power rating 5 , at investment time point 3 . The elements of $\tilde{C}$ need to be defined for each optimization time point $t$. As future expenses are worth less in today's currency, the elements of $\tilde{C}$ are depreciated over time using (2) and (3), $q$ being a user defined discount rate. The costs corresponding to the flow variables are set to zero (4).

$$
\begin{aligned}
\tilde{C} & =\left[C_{i, j, t=0}, C_{i, j, t=1}, \ldots, C_{i, j, t=N_{t}}\right] \\
C_{i, j, t=x} & =C_{i, j, t=0} \cdot(1-q)^{x}, \quad 1 \geq q \geq 0 \\
C_{i, j, t} & =0 \quad \forall U_{i j t}^{+}, U_{i j t}^{-}
\end{aligned}
$$

As $q$ is greater than zero, future costs are always lower than costs at the beginning of the planning horizon. Thus, more expensive investments are postponed as long as possible, as they are depreciated more. The expensive investments can only be postponed as long as the optimization constraints (5) - (15) allow it.

$$
\begin{aligned}
P_{t}^{\text {inter }, m, n} \leq & \left(U^{i n v}+U^{+}+U^{-}\right): \mathbf{l} \otimes \mathbf{k} \\
& \forall t \in\left[1, N_{t}\right], \forall m, n \in Z \\
P_{t}^{i m p, m} \leq & \left(U^{i n v}+U^{+}+U^{-}\right): \mathbf{1}_{\mathbf{k}}^{\mathbf{i m p}} \otimes \mathbf{k}, \\
& \forall t \in\left[1, N_{t}\right], \forall m \in Z \\
P_{t}^{\text {exp }, m} \leq & \left(U^{i n v}+U^{+}+U^{-}\right): \mathbf{1}_{\mathbf{k}}^{\exp } \otimes \mathbf{k}, \\
& \forall t \in\left[1, N_{t}\right], \forall m \in Z \\
P_{M P I C, t} \geq & \tilde{\Delta}_{M P I C}^{\text {ot }} \cdot\left(U^{i n v}+U^{+}+U^{-}\right) \cdot \mathbf{k}, \quad \forall t \in\left[1, N_{t}\right](8) \\
\overline{\mathbf{C}}_{\mathbf{t}} \geq & \left(U^{i n v}+U^{+}+U^{-}\right):(\overrightarrow{1} \otimes \mathbf{k}) \cdot \tilde{C}, \quad \forall t \in\left[1, N_{t}(9)\right. \\
0= & U_{d l}^{i n v}+U_{d l}^{+}+U_{d l}^{-} \forall t \leq \mathbf{t}_{\mathrm{dl}}, \\
\overrightarrow{1} \geq & E_{h v d c} \cdot\left(U_{h v d c}^{i n v}+U_{h v d c}^{+}+U_{h v d c}^{-}\right), \\
K_{i} \leq & \bar{K}, \forall i \in\left[1, t_{b}\right] \\
1= & \sum_{j, t} U^{i n v}, \forall i \in\left[1, N_{b}\right] \\
1= & \sum_{j, t} U^{+}, \forall i \in\left[1, N_{b}\right] \\
1= & \sum_{j, t} U^{-}, \forall i \in\left[1, N_{b}\right]
\end{aligned}
$$

Constraint (5) states that the sum of power ratings of tie lines between zones must be greater or equal than the desired interconnection power $P_{t}^{\text {inter, } m, n}$ for each time step of the 
planning horizon. The interconnection capacity needs to be fulfilled for each pair $m, n$ of zones separately. $Z$ is the set containing all independent transmission zones. $l$ is a vector indicating tie lines between the considered zones as defined in (16) and $\mathrm{k}$ is the vector of possible power ratings.

Constraints (6) and (7) state that the additional import and export capacity of each zone $m$ must be greater than the desired import/export capacity $P_{t}^{i m p, m}, P_{t}^{e x p, m}$. The reason of using these constraints is to consider transmission zones which can only import or export power. An example of such a zone is a large cluster of offshore wind farms. This way, inter-connectors between the offshore zone and all other zones can be built without specifying how much power needs to be imported in one particular region. This is determined depending on the power injection capabilities. $\mathrm{l}_{\mathrm{m}}^{\mathrm{imp}}$ and $\mathrm{l}_{\mathrm{m}}^{\mathrm{exp}}$ are incidence matrices similar to $\mathbf{l}$ defining which inter-connectors enable import/export to zone $m$.

$$
\mathbf{l}=\left\{\begin{array}{cc}
1 & \text { if branch } i \text { is a tie line } \\
0 & \text { otherwise }
\end{array}\right.
$$

Constraint (8) states that the maximum power injection capabilities of candidate nodes may not be exceeded if new connections are established. $\tilde{\Delta}_{M P I C}^{t o t}$ is defined as a $\left(N_{N} \cdot N_{t}\right) \times\left(N_{b} \cdot N_{k} \cdot N_{t}\right)$ matrix, $N_{N}$ being the number of candidate nodes. The elements of $\tilde{\Delta}_{M P I C}^{t o t}$ contain the matrices $\Delta_{M P I C}^{t o t}$ which indicate the change in the injection capabilities of candidate nodes depending on the power ratings of possible branches at each time point (17). The size of $\Delta_{M P I C, t}^{t o t}$ is $N_{N} \times\left(N_{b} \cdot N_{k}\right)$.

$$
\tilde{\Delta}_{M P I C}^{t o t}=\left[\begin{array}{cccc}
\Delta_{M P I C, t=0}^{t o t} & 0 & \cdots & 0 \\
\Delta_{M P I C, t=0}^{t o t} & \Delta_{M P I C, t=1}^{t o t} & \cdots & 0 \\
\vdots & \vdots & \vdots & \vdots \\
\Delta_{M P I C, t=0}^{t o t} & \Delta_{M P I C, t=1}^{t o t} & \cdots & \Delta_{M P I C, t=N_{t}}^{t o t}
\end{array}\right]
$$

Constraint (9) takes the limitation of capital availability into account and states that the sum of investments in a particular time step may not be higher than a defined maximum investment $\bar{C}_{t}$. The $1 \times N_{t}$ vector $\overline{\mathbf{C}}_{\mathbf{t}}$ contains the maximum investments per term which can have different values at each investment time point (18). By varying the elements of $\overline{\mathbf{C}}_{\mathbf{t}}$, several different investment sequences and grid configurations can be obtained.

$$
\overline{\mathbf{C}}_{\mathbf{t}}=\left[\overline{\mathbf{C}}_{1}, \overline{\mathbf{C}}_{2}, \ldots, \overline{\mathbf{C}}_{\mathrm{N}_{\mathrm{t}}}\right]
$$

It is important to analyse the effect of possible delays on the final transmission grid topology and the resulting costs in order to identify priority corridors. Constraint (10) includes possible delays in the optimization model. The constraint states that certain transmission paths can only be built after a pre-defined time point of the planning horizon. $U_{d l}^{i n v}, U_{d l}^{+}$and $U_{d l}^{-}$are a sub-set of the binary decision variables $U^{i n v}, U^{+}$and $U^{-}$ respectively, defining transmission paths facing delays. The vector $\mathbf{t}_{\mathbf{d l}}$ assigns time points to each element of the sub-sets

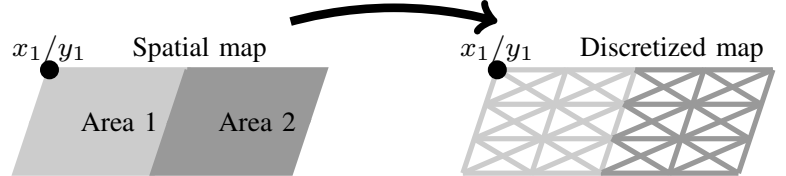

Figure 3: Creation of a weighted directed graph by discretizing a spatial map [6]

$U^{i n v}, U_{d l}^{+}$and $U_{d l}^{-}$from where on connections on these paths may be established.

Depending on technology availability, the final grid topology and the final investment costs can be different. Constraint (11) has been introduced in the problem formulation to analyse the effect of availability of meshed HVDC configurations. The constraint states that for all time points $t$ which are smaller than a defined time point $t_{H V D C}$ no multi-terminal or meshed HVDC configuration is possible. $U_{h v d c}^{i n v}, U_{h v d c}^{+}$and $U_{h v d c}^{-}$ indicate the decision variables of paths which are built in HVDC and $E_{h v d c}$ is an incidence matrix. This way only one HVDC link can be connected to candidate nodes before the time step $t_{H V D C}$.

Constraint (12) states that the maximum power rating of a connection path may not exceed a pre-defined maximum rating $\bar{K}$. Constraints (13) - (15) state that an investment in a candidate branch can only be made once.

\section{B. Optimal routing algorithm}

As the number of available technology options is advancing, nowadays it is not sufficient to consider only overhead lines for transmission grid expansion. Due to increased public opposition, permissions to build overhead lines (OHL) are hard to obtain [9]. When it comes to AC underground cables (UGC), the possible transmission distance is limited due to the high cable capacitance and the resulting charging currents. Therefore, the possibility of using HVDC transmission needs to be considered as well. Additionally, losses, being a considerable part of the life cycle costs depend on the chosen technology.

The transmission route and technology are interdependent. Additionally, the installation costs for different technologies depend on the type of soil, land acquisition costs and other factors. It is therefore important to optimize the cabling option (OHL vs. UGC), technology (HVAC vs. HVDC) and the transmission route at the same time. In the developed methodology, the optimization of technology and routing is defined around a weighted graph. Therefore, the considered installation area containing all candidate nodes is discretized to a grid of spatial points $p_{i}=\left[x_{i}, y_{i}\right]$ of size $N_{x} \times N_{y}$ as shown in Fig. 3. The number of chosen nodes and their horizontal and vertical position depend on the desired spatial resolution and the size of the area. All points $p_{i}=\left[x_{i}, y_{i}\right]$ are distributed in equal distance from each other.

A separate discretization is performed in order to represent the four technologies (AC OHL, AC UGC, DC OHL and DC UGC). Thus, the optimal routing and technology problem is formulated around a weighted graph $\mathcal{G}=(\mathcal{V}, \mathcal{E})$ having four technology layers and weighted edges signifying costs. 


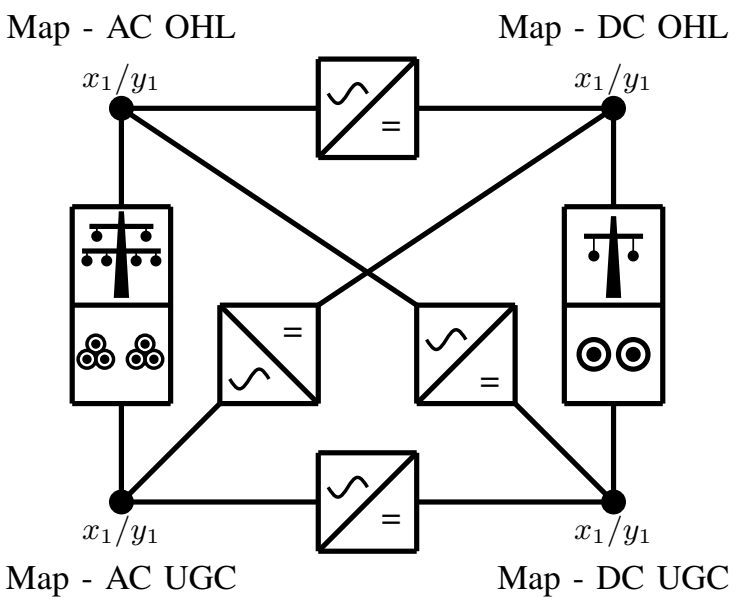

Figure 4: Creation of a weighted directed graph containing four technologies as input for the shortest path algorithm [5]

A full set of edges $\mathcal{E}$ between vertices $\mathcal{V}$ associated with adjacent spatial points correspond to cables or lines of a given technology. As fig. 4 shows, the edges between the "technology layers" correspond to a technology switch (e.g. an HVDC converter to switch from AC to DC or vice-versa).

The graph weight (cost) function $\mathcal{W}\left(p_{i}, \mathcal{E}_{j}\right)$ can take on a different positive value for every edge $j$ and represents the cost associated with progressing spatially or switching between technologies:

$\mathcal{W}\left(p_{i}, \mathcal{E}_{j}\right)=\left(c^{i n v}\left(p_{i}, \mathcal{E}_{j}\right)+c^{i n s t}\left(\mathcal{E}_{j}\right) \cdot w\left(p_{i}\right)\right) d\left(\mathcal{E}_{j}\right)+w^{\text {switch }}\left(\mathcal{E}_{j}\right)$

where $c^{i n v}\left(p_{i}, \mathcal{E}_{j}\right)$ and $c^{i n s t}\left(\mathcal{E}_{j}\right)$ correspond to both location and technology dependent investment and installation costs, respectively. $w\left(p_{i}\right)$ is a spatial weighting factor to take the change of installation costs in different areas into account. $d\left(\mathcal{E}_{j}\right)$ is the distance associated with the edge, and $w^{\text {switch }}\left(\mathcal{E}_{j}\right)$ is a technology conversion cost (e.g. HVDC converter station). The used costs depend on the optimal capacity $K$ determined by the other sub-problem, but is fixed for a given iteration. Additional cost components included in the spatial weight $\mathcal{W}\left(p_{i}, \mathcal{E}_{j}\right)$ are, reactive power compensation for $\mathrm{AC}$ connections, possible offshore platform costs and costs for connection of non-synchronous areas (back-to-back converters). The transmission losses are calculated for each technology option in dependence of the power rating using a standard load profile for all interconnectors. The costs of the losses are discounted according to the investment time point and added to the costs of equipment.

Including the above mentioned costs into a weighted graph, the shortest distance between the starting point and the termination point of a path can be determined using shortest path algorithms. If the weights assigned to the edges of the graph are costs per $\mathrm{km}$ of transmission line, the shortest path algorithm delivers the minimum transmission system cost between two vertices (20).
As all four technology layers are interconnected, the shortest path algorithm delivers the minimum transmission system cost

$$
\min _{S} C_{\text {equip }}=\sum_{\mathcal{E} \in \mathcal{S}} \mathcal{W}(\mathcal{E})
$$

where $S$ is a sequence of edges corresponding to the lowest cost or "shortest" path. Different shiortest path algorithms can be used to find the optimal route. In this work the heuristic $A^{*}$ algorithm has been used which has proven to be computationally more efficient [10] than Dijkstra's shortest path algorithm [11].

\section{Update of costs}

As shown in fig. 2, the optimal routing algorithm is only applied to paths selected by the MILP optimization. As the costs used in the initial MILP optimization are an approximation, the costs need updated in the following iterations. This is done by using the minimum costs obtained by the optimal routing algorithm. In order to avoid that the algorithm gets stuck in a local optimum, penalty functions are used for branches and power ratings which have not been selected. The following penalty functions are used

$$
c_{N S}^{z+1}= \begin{cases}c_{N S}^{z} \cdot\left(1-\overline{c_{\text {diff }}}\right)^{(3 / 4)} & \text { if } \overline{\overline{c_{\text {diff }}} \geq 0} \\ c_{N S}^{z} \cdot\left(1+\overline{c_{\text {diff }}}\right)^{(3 / 4)} & \text { if } \overline{c_{\text {diff }}}<0\end{cases}
$$

where $c_{N S}^{z}$ and $c_{N S}^{z+1}$ are the costs of not selected branches in iterations $z$ and $z+1$ respectively, and $\overline{c_{\text {diff }}}$ is the average of the relative cost differences between iterations $z$ and $z-1$ for the branches selected in iteration $z$. For power ratings and branches selected by the MILP optimization, the "true costs" obtained by the optimal routing algorithm is used. The iterative solution of the MILP and optimal routing problem is repeated until the relative cost difference $c_{\text {diff }}$ between two iterations is below a certain threshold $\epsilon$ or a maximum number of iterations have been reached. For the calculations shown in this paper, $\epsilon$ is defined as $10^{-5}$.

\section{CASE STUDY}

\section{A. Assumptions and input data}

In order to demonstrate a possible application of the developed methodology, it is applied to a possible North Sea region. The grid, generation and load data to calculate the maximum power injection capabilities are obtained from public sources. The French, German, Belgian and Dutch grid data is obtained from the websites of the according TSOs [12]-[16]. Based on the calculated maximum power injection capabilities (MPICs), 35 candidate nodes have been chosen in the CWE region. For the British grid, a reduced network of 29 nodes is used which is obtained from [17] where 15 candidate notes have been chosen based on the MPICs. The 32 node Nordic system is used to represent the Scandinavian grid [18] where 10 candidate notes have been selected. In this case it needs to be mentioned that the representations of the British and Nordic grid are not ideal for planning purposes and therefore the results need to be interpreted carefully. 
TABLE I

\begin{tabular}{lcc|cc|cc}
\multicolumn{9}{c}{ USED SPATIAL WEIGHTS } & \multicolumn{2}{c}{ Strategy 3 } \\
Areas & \multicolumn{2}{c}{ Strategy 1 } & \multicolumn{2}{c}{ Strategy 2 } & \multicolumn{2}{c}{ Stren } \\
& OHL & UGC & OHL & UGC & OHL & UGC \\
\hline Urban Areas & 40 & 2.5 & 40 & 2.5 & 100 & 2.5 \\
Mountains & 15 & 4.5 & 40 & 4.5 & 100 & 4.5 \\
Natural areas & 5 & 2 & 40 & 2 & 100 & 2 \\
Agricultural areas & 1 & 1 & 40 & 1 & 100 & 1 \\
Protected areas & 40 & 40 & 40 & 40 & 100 & 40 \\
Existing line corridors & 0.5 & 0.8 & 0.1 & 0.8 & 1 & 0.8 \\
Existing roads & 0.5 & 0.8 & 0.1 & 0.8 & 1 & 0.8 \\
Existing railways & 0.5 & 0.8 & 0.1 & 0.8 & 1 & 0.8 \\
Sea & 1000 & 0.75 & 1000 & 0.75 & 1000 & 0.75 \\
\hline
\end{tabular}

TABLE II

\begin{tabular}{|c|c|c|c|c|}
\hline \multicolumn{5}{|c|}{ DESIRED INTERCONNECTION CAPACITY } \\
\hline Zones & & $\begin{array}{c}P_{t}^{\text {inte }} \\
\text { year } 10\end{array}$ & $\begin{array}{l}\text { in MW } \\
\text { year } 20\end{array}$ & year 30 \\
\hline $\mathrm{GB} \rightarrow \mathrm{CWE}$ & 1500 & 3000 & 4500 & 6000 \\
\hline $\mathrm{CWE} \rightarrow \mathrm{GB}$ & 1500 & 3000 & 4500 & 6000 \\
\hline $\mathrm{SC} \rightarrow \mathrm{CWE}$ & 1000 & 2000 & 3000 & 3000 \\
\hline $\mathrm{CWE} \rightarrow \mathrm{SC}$ & 1000 & 2000 & 3000 & 3000 \\
\hline $\mathrm{NS} \rightarrow \mathrm{GB} / \mathrm{CWE} / \mathrm{SC}$ & 12000 & 25000 & 37000 & 50000 \\
\hline
\end{tabular}

The used spatial data covers existing infrastructure (rail roads, highways, existing $400 \mathrm{kV}$ grid), agricultural areas, mountains, nature areas (e.g. forests), Natura2000 protected areas and urban areas [19]. The spatial data has a resolution of $2.5 \mathrm{~km}$, hence the shown routes are indicative. In case detailed routes are desired, the algorithm needs to be applied to more accurate data with finer resolution. Tab. I shows the used spatial weights for the calculation of optimal routes.

Four different zones have been defined, being Great Britain (GB), Central West Europe (CWE), Scandinavia (SC) and the North Sea region (NS). Table II shows the desired additional transmission capacities between the zones. The chosen interconnection capacities are based on [20], [21]. It is assumed that the power from the North Sea region can be imporeted to any of the zones as long as the maximum power injection capabilities allow it. Four investment time points of 10 years are assumed.

\section{B. Calculation results}

Fig. 5 shows the optimal invetsment plan if links with a maximum power rating of $2 \mathrm{GW}$ can be used. In this case we can observe that most submarine connections are built using HVDC cables and onshore connections are built using HVDC overhead lines. In the first time step (fig. 5a) an AC submarine connection using a back-to-back converter is used to connect Belgium and the UK. In time step 2 (fig. 5b) an AC overhead line is proposed as a reinforcement between Belgium and France. In the remaining years the capacity of the links to connect wind farm clusters as well as interconnectors are increased. In the first and last step of the planning horizon interconnectors via offshore wind farm clusters are proposed.

Tab. III shows that the costs of losses account for $29.9 \%$ of the total lifecycle costs. Here it needs to be mentioned that the costs are calculated for a lifetime of 50 years, thus exceeding
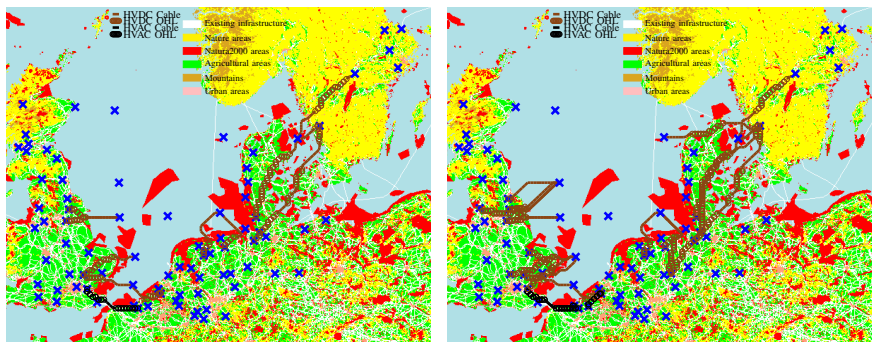

(a) Optimal grid expansion $t=0$ (b) Optimal grid expansion $t=10$ years

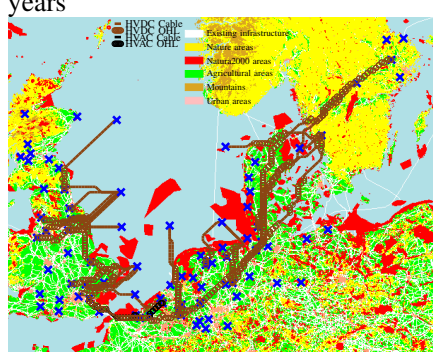
years

(c) Optimal grid expansion $t=20$ (d) Optimal grid expansion $t=30$ years years

Figure 5: Optimal investment plan for $\overline{\mathbf{K}}=2 G W$

\section{TABLE III}

\begin{tabular}{|c|c|c|c|c|c|c|c|c|}
\hline \multicolumn{9}{|c|}{ RATINGS AND $t_{H V D C}$} \\
\hline$t_{H V D C}$ & & $\overline{\mathbf{K}}=$ & $2 G W$ & & & $\overline{\mathbf{K}}=$ & $4 G W$ & \\
\hline 乎 & $\begin{array}{l}C_{t o t} \\
\mathrm{M} €\end{array}$ & $\begin{array}{c}C_{l o s s} \\
\mathrm{M} €\end{array}$ & $l_{\mathrm{km}}^{l_{O H L}}$ & $\begin{array}{c}l_{U G C} \\
\mathrm{~km}\end{array}$ & $\begin{array}{l}C_{t o t} \\
\mathrm{M} €\end{array}$ & $\begin{array}{c}C_{\text {loss }} \\
\mathrm{M} €\end{array}$ & $l_{\mathrm{km}}^{l_{\mathrm{H} L}}$ & $\begin{array}{l}l_{U G C} \\
\mathrm{~km}\end{array}$ \\
\hline 0 years & 3155 & 958 & 6711 & 7313 & 3081 & 896 & 3494 & 4182 \\
\hline 10 years & 3177 & 941 & 7003 & 7138 & 3092 & 910 & 4008 & 3977 \\
\hline 20 years & 3429 & 1016 & 8177 & 7157 & 3252 & 978 & 4191 & 3842 \\
\hline
\end{tabular}

the planning horizon. We can see that the total life cycle costs increase if multi-terminal HVDC connections are delayed.

Fig. 6 and 7 show the optimal investment plan if transmission paths of maximum $4 \mathrm{GW}$ can be used. The figures show that the number of necessary links drop in case larger links can be built. We can also see that stronger nodes further away from the coast are connected with longer and larger links. Tab. III compares the length of the transmission system for the analyzed cases. We can see that the total length of the transmission system decreases significantly if larger links can be used. The table shows that the total life cycle costs of the system are decreased by using links with higher power ratings.

\section{CONCLUSION}

In this paper a transmission system investment optimization methodology is demonstrated. The methodology determines the economically best expansion option between multiple transmission zones and takes spatial properties into account as well as different technology options. The developed methodology delivers a step-wise investment plan as output.

The paper shows how the methodology can be applied practically. It is improtant to mention that the results shown in this paper are based on public available data and do not reflect necessarily the real implemention of the system. In order to 

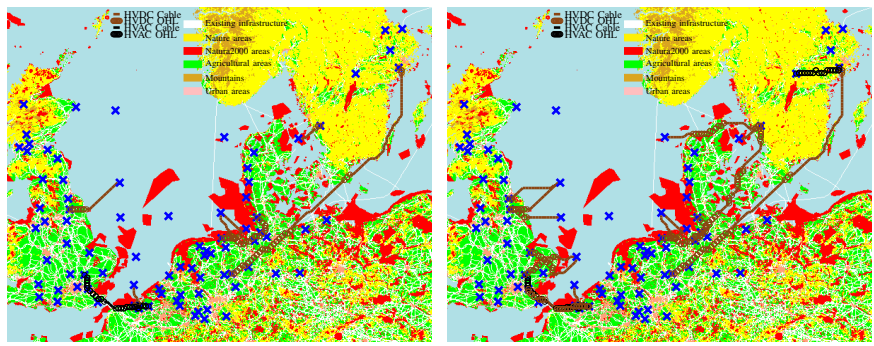

(a) Optimal grid expansion $t=0$ (b) Optimal grid expansion $t=10$ years

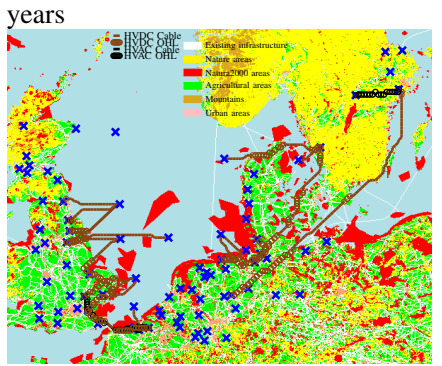
years

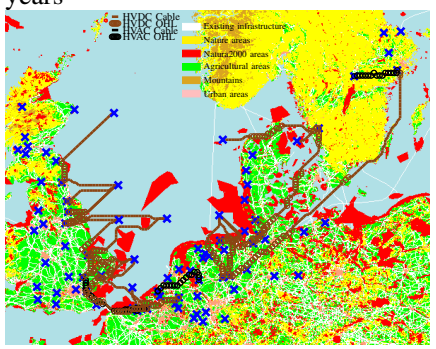

(c) Optimal grid expansion $t=20$ (d) Optimal grid expansion $t=30$ years

years

Figure 6: Optimal investment plan for $\overline{\mathbf{K}}=4 G W$
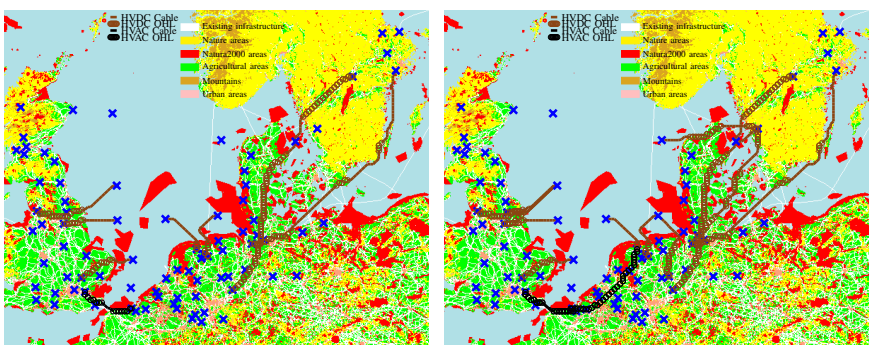

(a) Optimal grid expansion $t=0$ (b) Optimal grid expansion $t=10$ years

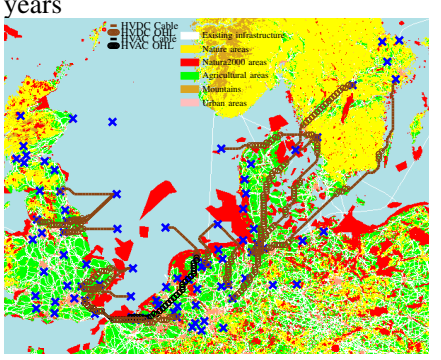
years

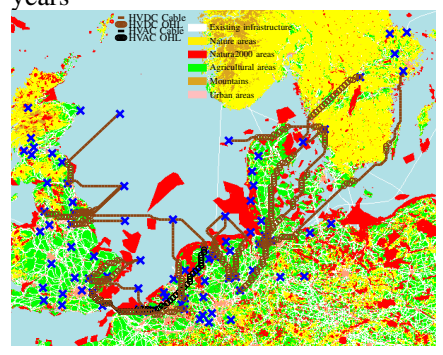

(c) Optimal grid expansion $t=20$ (d) Optimal grid expansion $t=30$ years years

Figure 7: Optimal investment plan for $\overline{\mathbf{K}}=4 G W$ and multi-terminal HVDC possible after 10 years

obtain more relaible results, more accurate data on network, power flows, costs and spatial properties is needed.

The developed methodology relies on integer programming to solve the optimization problem. This causes long computation times and convergence problems as many commercial and open source solvers still cannot achieve the same performance as for continious variables especially for a large number of optimization variables. In future, the developed methodology needs to be improved further to be computationally more efficient and thus applicable to even larger systems.

\section{ACKNOWLEDGMENT}

This work is supported by ABB, Elia and Vattenfall in the framework of the industrial research project "Reaching for a Pan-European Grid: How to Make the Transition? Transmission System Investment Planning".

\section{REFERENCES}

[1] Entso-e. Ten Year Network Development Plan 2012 https://www.entsoe.eu/fileadmin/user_upload/_library/SDC/TYNDP/ 2012/TYNDP 2012 report.pdf.

[2] G. Latorre, R. Cruz, J. Areiza, and A. Villegas, "Classification of Publications and Models on Transmission Expansion Planning," Power Systems, IEEE Transactions on, vol. 18, no. 2, pp. 938 - 946, may 2003.

[3] C. Lee, S. Ng, J. Zhong, and F. Wu, "Transmission expansion planning from past to future," in Power Systems Conference and Exposition, 2006. PSCE '06. 2006 IEEE PES, Oct 2006, pp. 257-265.

[4] A. Conejo, E. Castillo, R. Minguez, and R. Garcia-Bertrand, Decomposition Techniques in Mathematical Programming - Engineering and Science Applications, 1st ed. Springer-Verlag, 2006, ISBN 978-3-540-27685-2.

[5] H. Ergun, B. Rawn, R. Belmans, and D. Van Hertem, "Technology and Topology Optimization for Multizonal Transmission Systems," Power Systems, IEEE Transactions on, vol. 29, no. 5, pp. 2469-2477, Sept 2014.

[6] — "Stepwise Investment Plan Optimization for Large Scale and Multi-Zonal Transmission System Expansion," Power Systems, IEEE Transactions on, accepted for publication, 2015.

[7] H. Ergun, D. Van Hertem, and R. Belmans, "Identification of Power Injection Capabilities for Transmission System Investment Optimization,' 10th IEEE PowerTech Conference, June 2013, Grenoble, France.

[8] Hakan Ergun, "Grid Planning for the Future Grid, Optimizing Topology and Technology Considering Spatial and Temporal Effects," Ph.D. dissertation, University of Leuven, Faculty of Engineering Science, January 2015

[9] S. P. Vajjhala and P. S. Fischbeck, "Quantifying siting difficulty: A case study of us transmission line siting," Energy Policy, vol. 35, no. 1, pp. $650-671,2007$

[10] R. Dechter and J. Pearl, "Generalized Best-first Search Strategies and the Optimality of A*," J. ACM, vol. 32, no. 3, pp. 505-536, Jul. July 1985.

[11] E. Dijkstra, "A Note on Two Problems in Connexion with Graphs," Numerische Mathematik, vol. 1, no. 1, pp. 269-271, 1959.

[12] RTE France. (2015) Static Grid Model. https://clients.rte-france.com/ lang/an/visiteurs/vie/indispos_caracteristiques_statiques.jsp. Last checked: 28.09.2014

[13] Elia. (2015) Technical Grid Data. http://www.elia.be/en/grid-data/ Grid-Technical-Data. Last checked: 28.09.2014.

[14] Tennet. (2015) Statisches Netzmodell. http://www.tennettso de/site/Transparenz/veroeffentlichungen/statisches-netzmodell/ statisches-netzmodell. Last checked: 28.09.2014.

[15] Ampiron. (2015) Statisches Netzmodell. http://www.amprion.net/ statisches-netzmodell. Last checked: 28.09.2014.

[16] Transnet BW. (2015) Standards + Zukunft. https://www.transnetbw. de/de/strommarkt/engpassmanagement/standards-zukunft. Last checked: 28.09.2014.

[17] University of Edinburgh. (2015) Power Systems Test Case Archive. http: //www.maths.ed.ac.uk/optenergy/NetworkData/reducedGB/. Last checked: 28.09.2015

[18] "Nordic32A A Cigre test system for simulation of transient stability and long term dynamics," K. Walve, Tech. Rep., 1994.

[19] H. Ergun, B. Rawn, R. Belmans, and D. Van Hertem, "Optimization of transmission technology and routes for Pan-European electricity Highways considering spatial aspects," in PowerTech, 2015 IEEE Eindhoven, June 2015, pp. 1-6.

[20] "Offshore Grid Development in the North Seas ENTSO-E views," Entso-e, Tech. Rep., 2011. [Online]. Available: http://www.eurelectric. org/Download/Download.aspx?DocumentFileID=71840

[21] S. Torbaghan, M. Gibescu, B. Rawn, and M. Van Der Meijden, "A Market-Based Transmission Planning for HVDC Grid - 2014;Case Study of the North Sea," Power Systems, IEEE Transactions on, vol. 30, no. 2, pp. 784-794, March 2015. 